\title{
In-Situ Mass Thickness Calibrations Using MWCNTs
}

\author{
M. Malac ${ }^{1,2}$, D. Homeniuk ${ }^{1}$, M. Hayashida ${ }^{1}$, T. Fujii ${ }^{3}$, T. Yaguchi ${ }^{3}$, R.F. Egerton ${ }^{2}$ \\ ${ }^{1 .}$ NRC-NANO, Edmonton, Canada. \\ 2. Department of Physics, University of Alberta, Edmonton, Canada. \\ ${ }^{3 .}$ Hitachi High-Technologies Corp., Hitachinaka-shi, Ibaraki-ken, Japan.
}

Bright field transmission electron microscope (BFTEM) images are often used to measure sample size in two dimensions (2D). Mass thickness ( $\rho t$ ) maps of the sample along the electron beam direction, although measurable with a monolayer precision $[1,2]$, are seldom obtained. To measure $\rho t$, a calibration sample is needed. We show that calibration can be obtained in-situ using multi wall carbon nanotubes (MWCNTs).

BFTEM contrast $C$ for the purpose of $\rho t$ measurement is defined as $C=\log \left(I_{0} / I_{\mathrm{tr}}\right)$ and is proportional to $(\rho t)$.

$C=\log \left(I_{0} / I_{\text {tr }}\right)=N_{\mathrm{a}} A^{-1} \sigma \rho t$

Here $I_{\text {tr }}$ is the intensity transmitted through a sample and the subsequent angle-limiting aperture. $I_{0}$ is the corresponding incident intensity in vacuum, $N_{\mathrm{a}}$ is the Avogadro number, $A$ is their atomic weight and $\sigma$ is the scattering cross section to angles larger than the collection angle [1-3]. To relate $C$ to $\rho t$ in units of $\left[\mu \mathrm{g} / \mathrm{cm}^{2}\right]$, the contrast $C$ of objects with known $\rho t$ must be measured. MWCNTs have wall density of graphite $\left(\rho=2.266 \mathrm{~g} / \mathrm{cm}^{3}\right)$ and a core that is either hollow or contains other materials. The projected thickness of the MWCNT wall can be calculated, see Fig. 1, as function of distance $x$ from its center in the specimen plane. The projected $\rho t$ of MWCNT can be written as:

$\rho t=2 \rho \sqrt{R_{1}^{2}-x^{2}} \quad$ for $R_{2}<\mathrm{x}<R_{1}$

Here $R_{1}$ and $R_{2}$ are the external and internal radii of the MWCNT. Eq. (1) and (2) lead to

$C^{2}=\left(\mathrm{N}_{\mathrm{a}} \mathrm{A}^{-1} \sigma\right)^{2} 4 \rho^{2} R_{1}^{2}-\left(\mathrm{N}_{\mathrm{a}} \mathrm{A}^{-1} \sigma\right)^{2} 4 \rho^{2} x^{2}$

that is in the form $y^{2}=b-a x^{2}$. A linear fit to a plot of measured $C$ squared as function of $x^{2}$ for a MWCNT, see Fig 2, yields $a=\left(\mathrm{N}_{\mathrm{a}} \mathrm{A}^{-1} \sigma\right)^{2} 4 \rho^{2}$ that relates measured $C$ to $\rho t$, as $\rho t=2 * \log \left(I_{0} / I_{\mathrm{tr}}\right) / \operatorname{sqrt}(a)$, that can be evaluated at every pixel of an image, resulting in a $\rho t$ map. A fitted radius can be obtained as $R_{1}=$ $\operatorname{sqrt}(b / a)$.

Experiments were performed in a Hitachi HT7700 equipped with a Gatan Orius SC200W camera and W hairpin filament, operated at $100 \mathrm{kV}$. A $12.4 \mathrm{mrad}$ collection angle was defined by the objective aperture. A $19 \mathrm{~nm}$-wide contrast profile of a clean segment of a MWCNT was extracted and fitted in Fig 2 for the $x>0$ (red) and $x<0$ (blue) sections of the MWCNT. The slope $a=4.7 \times 10^{-4} \mathrm{~nm}^{-1}$ (blue) and $3.7 \times 10^{-4} \mathrm{~nm}^{-}$ ${ }^{1}$ (red) for the respective sections of the MWCNT fit.

Fig. 3 shows $\rho t$ profile obtained from $2 \log \left(I_{0} / I_{\mathrm{tr}}\right) / \operatorname{sqrt}(a)$ in blue and red, simulation using Eq. 1 and 2 , and fitted $R_{1}$ and $\mathrm{N}_{\mathrm{a}} \mathrm{A}^{-1} \sigma\left(R_{1}=11.2 \mathrm{~nm}\right.$ in purple for the $x>0$ region and $R_{1}=11.4 \mathrm{~nm}$ in yellow for $x<0$ region). Fig. 3 also shows simulated $\rho t$ using visually measured $R_{1}=14 \mathrm{~nm}$ and fitted $\mathrm{N}_{\mathrm{a}} \mathrm{A}^{-1} \sigma($ green). The fitted values of $R_{1}$ are an underestimate as compared to visual measurement of a BFTEM image, possibly due to presence of a Fresnel fringe at the edge of the MWCNT. The $\rho t$ obtained using fitted $\mathrm{N}_{\mathrm{a}}$ $\mathrm{A}^{-1} \sigma$ and experimental $C(x)$ in red and blue yields a good agreement with the expected $\rho t$ for visually measured $R_{1}$ (green). The mean difference of $\rho t$ over the MWCNT, indicated by an arrow in Fig. 3, is (- 
$0.2 \pm 0.3) \mu \mathrm{g} / \mathrm{cm}^{2}$. It indicates that the slope $a$ yields a reasonable estimate of $\mathrm{N}_{\mathrm{a}} \mathrm{A}^{-1} \sigma$, but the intercept $b$ underestimates $R_{1}$.

Fig. 4 shows an example 2D $\rho t$ obtained by the above method. Part of the MWCNT area marked by a green rectangle was fitted to obtain the calibration constants $a$ and $b$. Fig. 4. also illustrates the effect of diffraction contrast. To reduce the effect of diffraction contrast, a large collection angle should be used, although the large collection aperture reduces the slope $a$ and thus sensitivity of the method [1, 2, 4].

\section{References:}

[1] T Fujii et al., Microscopy and Microanalysis 24, p. 1612.

[2] H-R Zhang et al., Micron 43, p. 8.

[3] L Reimer, H Kohl, Transmission Electron Microscopy $5^{\text {th }}$ ed., Springer, 2008.

[4] We acknowledge Dr. Francisco Paraguay Delgado, CIMAV, Mexico, for kindly providing the MWCNT sample.

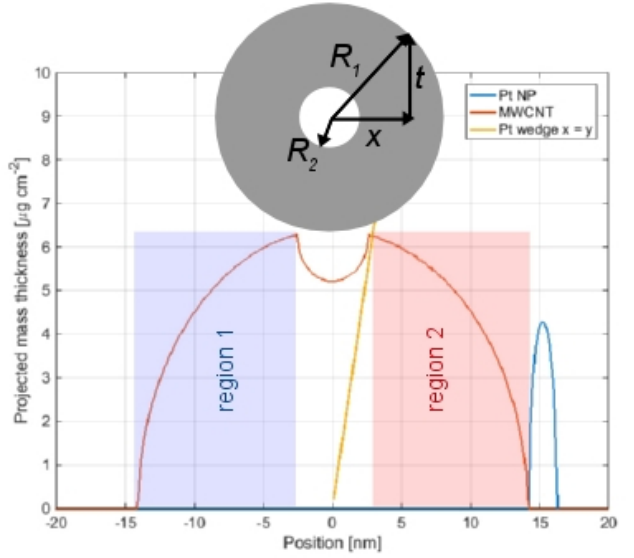

Fig 1. Simulated $\rho$ t of MWCNT (red) Pt NP (blue) and a $45^{\circ} \mathrm{Pt}$ wedge (yellow). The contrast is fitted over regions 1 and 2 separately. The blue region is flipped to the positive quadrant for the purpose of the fit.

A schematic drawing of MWCNT cross section with outer radius $R_{1}$ projected thickness $t$ is shown in grey. The yellow line can be used to interpret contrast of Pt NPs.

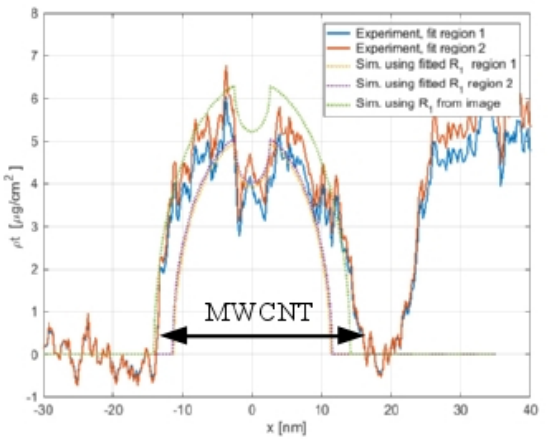

Fig 3. $p t$ of MWCNT using fitted $\mathrm{N}_{\mathbf{a}} \mathrm{A}^{-1} \sigma$ and experimental $C$ (red, blue) and simulations using Eq. 1 with $R_{1}$ from fit (yellow, purple) and $\mathrm{N}_{\mathrm{a}} \mathrm{A}^{-1} \sigma$ from fit and $\mathrm{R} 1$ obtained from visual measurement of inset in Fig. 2.

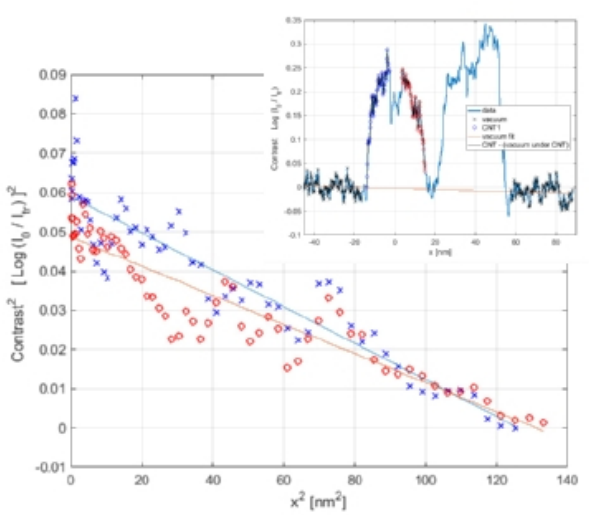

Fig 2. Contrast squared, $C^{2}$, plotted as function of square distance $x^{2}$ from the centre of the MWCNT A linear fit for region 1 and 2, as indicated in Fig 1 . allows to relate image contrast and known mass thickness of the MWCNT. Inset: raw contrast profile (see Fig. 4) and background removal

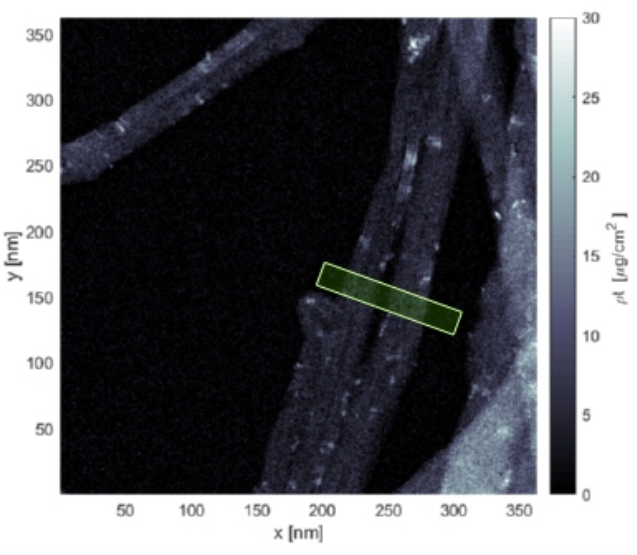

Fig 4. Mass thickness map obtained from a single BFTEM image complemented by a vacuum image as $\rho t=\log \left(I_{0} / I_{\mathrm{t}}\right) *\left(\mathrm{~N}_{\mathrm{a}} \mathrm{A}^{-1} \sigma\right)^{2}$. The calibration was obtained using the NP-free region of the MWCNT marked with a green rectangle. 